\title{
Microwave signal processing based on ultrafast dynamics in quantum dot waveguides
}

\author{
Chen, Yaohui; Mørk, Jesper
}

Published in:

International Conference on Transparent Optical Networks

Link to article, DOI:

10.1109/ICTON.2010.5549185

Publication date:

2010

Document Version

Publisher's PDF, also known as Version of record

Link back to DTU Orbit

Citation (APA):

Chen, Y., \& Mørk, J. (2010). Microwave signal processing based on ultrafast dynamics in quantum dot waveguides. In International Conference on Transparent Optical Networks (pp. 1-4). IEEE.

https://doi.org/10.1109/ICTON.2010.5549185

\section{General rights}

Copyright and moral rights for the publications made accessible in the public portal are retained by the authors and/or other copyright owners and it is a condition of accessing publications that users recognise and abide by the legal requirements associated with these rights.

- Users may download and print one copy of any publication from the public portal for the purpose of private study or research.

- You may not further distribute the material or use it for any profit-making activity or commercial gain

- You may freely distribute the URL identifying the publication in the public portal 


\title{
Microwave Signal Processing Based on Ultrafast Dynamics in Quantum Dot Waveguides
}

\author{
Yaohui Chen and Jesper Mork \\ DTU Fotonik, Department of Photonics Engineering, Technical University of Denmark, Lyngby, Denmark \\ Tel: (45) 4525 6352, Fax: (45) 4593 6581, e-mail:yach@fotonik.dtu.dk
}

\begin{abstract}
In this paper we review theoretical work on slow and fast light effects in quantum dot (QD) semiconductor waveguides and the potential applications in microwave photonics. In particular we emphasize the unique ultrafast carrier dynamics occurring between discrete QD bound states and its influence on the dynamic gain grating and cross gain modulation in QD semiconductor optical amplifiers (SOAs). The exploitation of ultrafast carrier dynamics enables the realization of phase shifters at frequencies in the range of $100 \mathrm{GHz}$.
\end{abstract}

Keywords: Quantum dots, semiconductor optical amplifier, microwave photonics.

\section{INTRODUCTION}

Controllable slow and fast light using coherent population oscillations (CPO) has been experimentally demonstrated in solid state crystals [1] and different active semiconductor waveguides at room temperature [2-4]. The change of light speed can be described as an effect of index dispersion (dynamical gain grating) arising from wave mixing in saturable absorption or gain media, where the population is driven coherently by the beating of two light beams. Such index dispersion with contributions from various carrier dynamical processes leads to a change in the relative amplitude and phase of the spectral components, which is also referred to as Bogatov effect in semiconductor materials [5]. So far carrier density pulsations (CDP) with carrier lifetime on the scale of tens of picoseconds to a nanosecond has been the dominating effect in experiments and modeling involving sinusoidally modulated optical signals in semiconductor optical amplifiers (SOAs) [2,3]. For potential applications in microwave photonics, it is of significant interest to use semiconductor devices as slow light element in realizing an optically fed microwave phase shifter. Great efforts towards alleviating the limitation of bandwidth as well as maximum variable phase shift range have been reported [6-9]. Recent calculations and experiments indicate that quantum dot (QD) based devices are good candidates for high speed optical signal processing due to the unique ultrafast inter-subband carrier dynamics between discrete QD bound states [9-13].

In this work, we review our theoretical work on ultrafast inter-subband carrier dynamics occurring between discrete QD bound states. In particular, we discuss the influence on the dynamic gain grating and cross gain modulation (XGM) in QD SOAs and their potential implementations as microwave phase shifter working at modulation frequencies in the range of $100 \mathrm{GHz}[14,15]$.

\section{CARRIER AND GAIN DYNAMICS IN SEMICONDUCTOR QUANTUM DOT WAVEGUIDE}

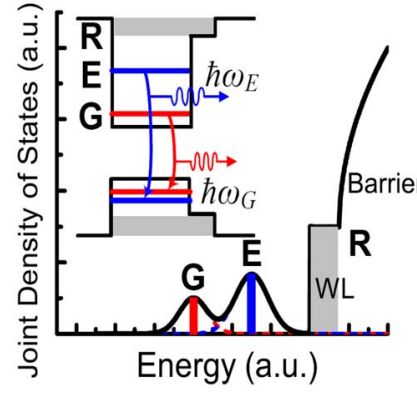

(a)

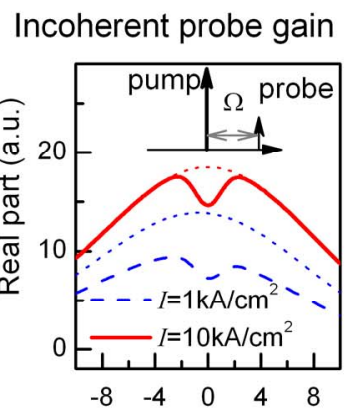

Frequency Detuning, $\Omega / 2 \pi$, (THz)

(b)

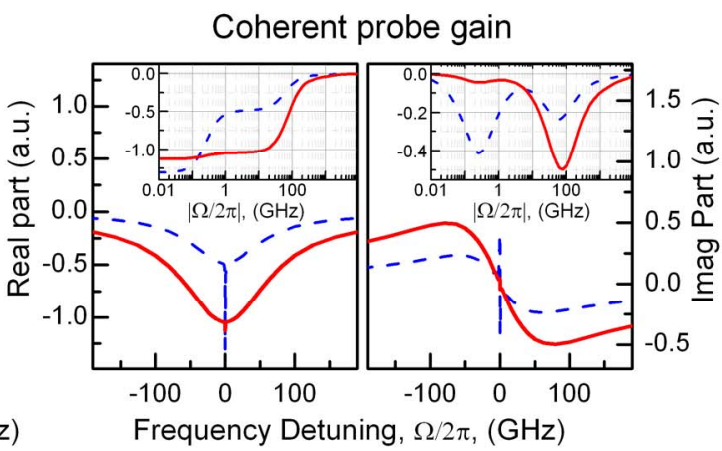

(c)

Fig. 1: (a) Schematic diagram of inhomogeneously broadened ODs. Examples of (b) incoherent and (c) coherent electric field probe gain in inhomogeneously broadened $Q D S$ versus probe-pump frequency detuning $(\Omega / 2 \pi)$ at two different injection currents. Zero linewidth enhancement factor is assumed. The pump is located at optical transition corresponding to discrete QD states. Dotted lines in (b) are the unsaturated gains at corresponding injection currents.

Our QD SOA model is based on the well-established rate equation approach originally developed for describing carrier dynamics in $1100 \mathrm{~nm}$ inhomogeneously broadened InAs/GaAs QDs [10], as shown in Fig. 1(a). Such model treats inhomogeneous (size) broadening by dividing the ensemble into subgroups of dots with similar size. In each dot subgroup, electrons/holes from a common reservoir, wetting layer (WL) and barrier, are assumed to only occupy the two lowest discrete QD bound states, i.e., ground (G) states and (E) states. Boltzmann scattering model based on Fermi's golden rule are used to describe the ultrafast electron inter-subband transitions between discrete QD states with pairs of capture and escape processes. The phenomenological rates for these 
capture/escape processes depend on the carrier density and temperature via phonon and Auger assisted contributions [10]. The typical characteristic times ranging from subpicoseconds to tens of picoseconds were extracted from two-color pump-probe measurements [16] and carrier capture calculations [17,18]. Due to the narrow spacing between valence band levels, which follows from the large hole mass, we have used a local carrier density description of the dynamics of the holes, with an intra-band scattering time of 100 fs. Nanosecond scale carrier lifetimes for reservoir are used.

Assuming zero linewidth enhancement factor (neglecting the refractive index dynamics), the gain dynamics corresponding to the discrete QD states can be evaluated by calculating the electric field probe gain as a function of frequency detuning in a simple continuous wave $(\mathrm{CW})$ pump-probe configuration. The incoherent part shown in Fig. 1(b) illustrates the gain saturation with a spectral hole burning (SHB) profile governed by a homogeneous linewidth around one THz. Meanwhile, the coherent part (dynamic gain grating, or referred as CPO effect) shown in Fig. 1(c) has two distinctive resonant contributions at tens of gigahertz and sub-gigahertz bandwidth, corresponding to ultrafast electron inter-subband dynamics (featured by electron capture processes for gain recovery) and slow total carrier density pulsation respectively. At low injection current, the sub-gigahertz resonance dominates the coherent response which is similar to CDP in the bulk material. At high injection current, the sub-gigahertz resonance is greatly suppressed and the enhanced wideband resonance enables high speed optical signal processing in QD devices [11]. When additional optical carriers are involved, similar ultrafast coherent gain dynamics can also be revealed as high speed XGM effects in QDs [12].

\section{MICROWAVE PHASE SHIFTER BASED ON QD SOAS}

\subsection{Results of coherent population oscillation (CPO) effect $^{[14]}$}

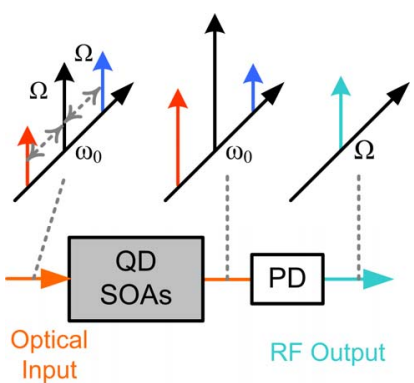

(a)

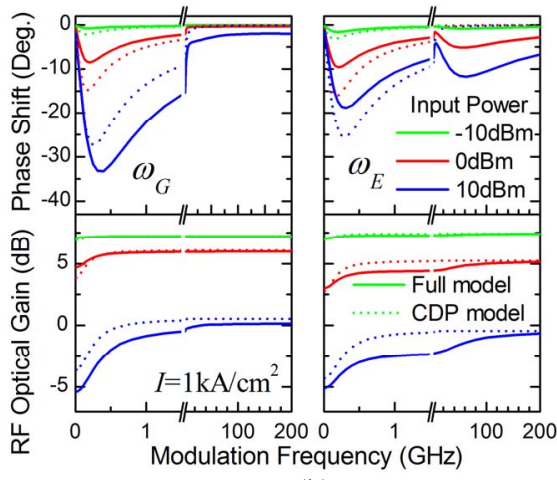

(b)

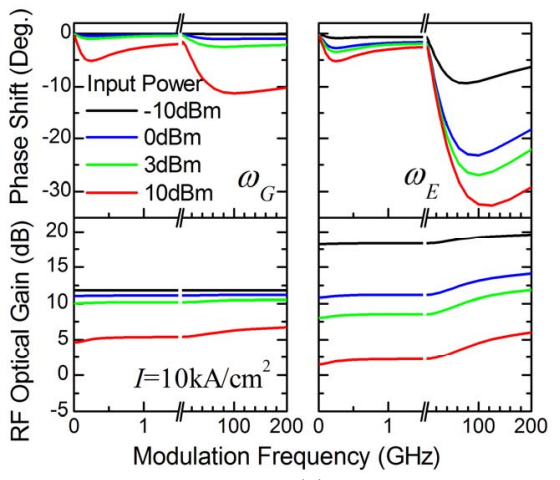

(c)

Fig. 2: (a) Schematic configuration for CPO based slow/fast light effects in QD SOAs. PD: photodetector. (b) Phase shift and RF optical gain as a function of modulation frequency at optical transitions corresponding to ground and excited states for different input pump powers under moderate injection current $\left(1 \mathrm{kA} / \mathrm{cm}^{2}\right.$ ) (fast light in gain regime). Solid curves are based on the full model. Dotted curves are based on the CDP model used in [3,4]. (c) The corresponding Phase shift and RF optical gain under strong injection current $\left(10 \mathrm{kA} / \mathrm{cm}^{2}\right)$.

Fig. 2(a) shows the conventional scheme to achieve slow/fast light based on CPO effects for sinusoidally modulated optical signals $[2-4,6]$. The slow/fast light effects are characterized via the phase shift that an RF modulated envelope undergoes and can be tuned either by controlling the bias of the QD SOAs or by varying the input optical power. In principle, both gain and refractive index in an SOA give important contributions to the amplitude and phase changes of different spectral components. However, since the phase change imposed on a probe component by wave mixing with a pump is anti-symmetric with respect to the detuning frequency between pump and probe, the effect of such anti-symmetric phase change cancels out for a conventional double sideband signal with a central carrier (pump) and two modulation sidebands (probes) [3]. In general, fast light (negative phase shift) is observed in the gain regime. Fig. 2(b) shows the simulated phase shift and RF optical gain as a function of modulation frequency at different optical transitions corresponding to ground state $\left(\omega_{G}\right)$ and excited state $\left(\omega_{E}\right)$ for different input pump powers. For the $\mathrm{G}$ transition, a maximum phase shift of around $-34^{\circ}$ is observed at a peak modulation frequency around $300 \mathrm{MHz}$ by increasing the input power by $20 \mathrm{~dB}$, and this phase shift is accompanied by a steep RF optical gain variation. As the modulation frequency is increased, the phase shifts decreases. These results agree well with experimental observations [4] and are explained by the slow CDP model $[3,4]$. As we consider the case of the E transition, we observe the appearance of phase shift maxima at two separate frequencies: a value of $-18^{\circ}$ peaked around $300 \mathrm{MHz}$ and a value of $-12^{\circ}$ peaked at a much higher frequency of around $70 \mathrm{GHz}$. Because of the different saturation powers for $\mathrm{G}$ and $\mathrm{E}$ transitions, even with similar modal gain, the magnitude of the two phase shifts differs considerably. We observe also that the two phase shift peaks correspond to the two different plateau-levels of RF optical gain seen in the lower plot of Fig. 2(b). The high-frequency peak corresponds to the resonant response determined by the ultrafast electron inter-subband dynamics as shown in Fig. 1(c). As we increase the injection current to $10 \mathrm{kA} / \mathrm{cm}^{2}$, this electron transition dynamics in QDs is further enhanced and dominates over the CDP mechanism. The second phase shift 
peak is enhanced and shifted towards $100 \mathrm{GHz}$, or even higher frequencies, as shown in Fig. 2(c). The plateaus of constant RF optical gain are also extended to higher frequencies. In reality, the ultrafast carrier dynamics in QD SOAs might strongly depend on the bias control condition and signal power as well as dot shape and fabrication process via the intersubband scattering times, see e.g. [19], which provides potential for controlling the second peak modulation frequency.

\subsection{Results of cross gain modulation (XGM) effect ${ }^{[15]}$}

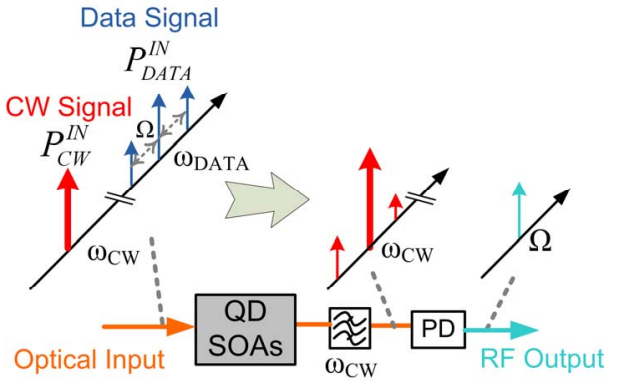

(a)

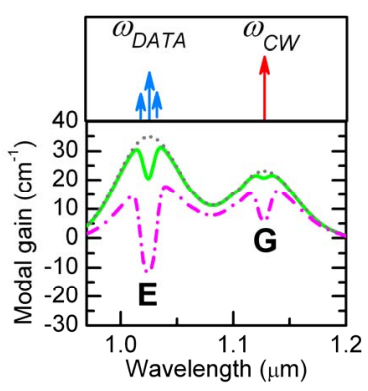

(b)

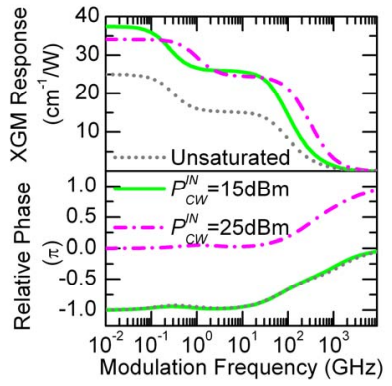

(c)

Fig. 3 (a) Configuration of wavelength up-conversion based on cross gain modulation in OD SOAs. (b) Calculated incoherent modal gain profile and (c) XGM response and relative phase for the up-conversion scheme in QD SOAs at a strong current density (10kA/cm ${ }^{2}$ ) for different input $C W$ power. Input data signal is set to $1 \mathrm{~mW}$ with $20 \%$ modulation index.

Alternatively, we also proposed a novel dual-wavelength-configured phase shifter based on dynamic gain gratings induced by high speed XGM in QD SOAs [15]. Fig. 3(a) shows the considered wavelength upconversion configuration based on QD SOAs. There are two main changes compared to the conventional small signal XGM configuration in [12]. First, the optical frequencies of the data signal $\left(\omega_{D A T A}=\omega_{E}\right)$ and $\mathrm{CW}$ signal $\left(\omega_{C W}=\omega_{G}\right)$ are chosen corresponding to the two lowest discrete QD bound states, i.e., excited (E) and ground $(\mathrm{G})$ state, which are connected by fast (sub-picosecond) inter-subband electron relaxation. The frequency detuning $\left(\omega_{D A T A}-\omega_{C W}=\omega_{E}-\omega_{G}\right)$ is assumed much larger than the homogenous linewidth of the QDs, and thus FWM interaction between data and $\mathrm{CW}$ signals are neglected. Secondly, the input $\mathrm{CW}$ power $P_{C W}^{I N}$ is variable and acts as a strong pump, while the average input data power $P_{D A T A}^{I N}$ is constant and relatively weak. Therefore the dynamic gain grating is no longer solely determined by the data signal as in the small signal regime. Instead, in terms of the two wave competition [20], both the data and XGM converted signal are considered to compete for the available carriers and interact with dynamic gain gratings (at frequencies $\omega_{D A T A}$ and $\omega_{C W}$ ) via fast intersubband effects in QDs, especially under a high current injection. By ideal flat-top selective optical bandpass filtering, we can characterize the output phase shift $\varphi_{X}^{O U T}$ of the intensity envelope at the given optical frequency $\omega_{X}(X=C W, D A T A)$. Fig. 3(b) shows the calculated incoherent modal gain of QD SOAs for different values of the input $\mathrm{CW}$ power. As the stimulated emission at frequency $\omega_{C W}$ (input $\mathrm{CW}$ power) increases, spectral holes are seen to develop in the gain spectrum, centered at the E and G states. Notice that the SHB corresponding to the $\mathrm{E}$ state transition originates from the large contrast between the fastest intradot electron relaxation and the intermediate electron capture from $\mathrm{R}$ to $\mathrm{E}$ states, which is synonymous to the existence of an injection bottleneck due to long capture time or short escape time [21]. As the rate of removal of carriers in the QD G state due to stimulated emission approaches the injection rate between reservoir and QDs, it is possible to deplete the E state carrier population and thus even switch from gain to absorption. Fig. 3(c) shows the corresponding coherent part of gain dynamics in XGM effect. Flat XGM responses approximately up to $100 \mathrm{GHz}$ are observed, which reveals the role of fast inter-subband QD carrier dynamics [11]. As the CW power is modest, the XGM responses in the low modulation frequency range have a phase shift of around $-\pi$ relative to the modulation of the input data signal, which is similar to wavelength conversion with an inverse pattern in the small signal regime [12]. As the $\mathrm{CW}$ power is strong and depletes the incoherent gain of $\mathrm{E}$ state into absorption, a $\pi$-shift of the XGM response is consistent with switching to "non-inverting" cross absorption modulation (XAM) [22]. Thus, by increasing the input CW power, the XGM converted signal experiences the corresponding $\pi$-shift and also benefits from the efficient conversion at high modulation frequencies.

Fig. 4(a) shows the calculated characteristics of the RF output signal at a modulation frequency of $40 \mathrm{GHz}$ in our wavelength conversion configuration under strong current injection. We fix the input data signal at $1 \mathrm{~mW}$ to retain a reasonable signal to noise level. The top part of Fig. 4(a) shows a $\sim 180$ degree tunable phase shift $\varphi_{C W}^{O U T}$ for the $X G M$ converted output by controlling the input probe power. The sharp increase of the phase shift corresponds to the notch-type drop of the XGM efficiency (related to the RF optical gain) seen in the bottom part of Fig. 4(a) at frequency $\omega_{C W}$. In the small signal regime, only the data signal dominates the dynamic gain grating and a linear increase of XGM efficiency can be observed. The intensity envelopes of the output data signal and the XGM converted signal are nearly out of phase $\left(\sim 180\right.$ degree for $\left.\varphi_{C W}^{O U T}-\varphi_{D A T A}^{O U T}\right)$. In the two-wave- 
competition regime, the dynamic gain gratings also depend on the mean power of the spatially varying $\mathrm{CW}$ signal. As the stimulated emission at the $\mathrm{G}$ state transition reaches the maximum value imposed by the injection bottleneck, the amplifiers can be regarded as being spatially divided into a usual XGM section and an XAM section. Thus the intensity envelope of the $X G M$ signal experiences a $\pi$-shift in between these two sections, which results in a notch-type drop in the XGM efficiency and a $\sim 180$ degree phase shift. As such $\pi$-shift is governed by the incoherent part of gain dynamics by two-wave competition, we can achieve similar 180 degree phase shifting properties at a broad bandwidth even beyond $100 \mathrm{GHz}$ as shown in Fig. 4(b). However, we also noticed that the corresponding RF optical gain changes significantly, which is an undesirable feature.

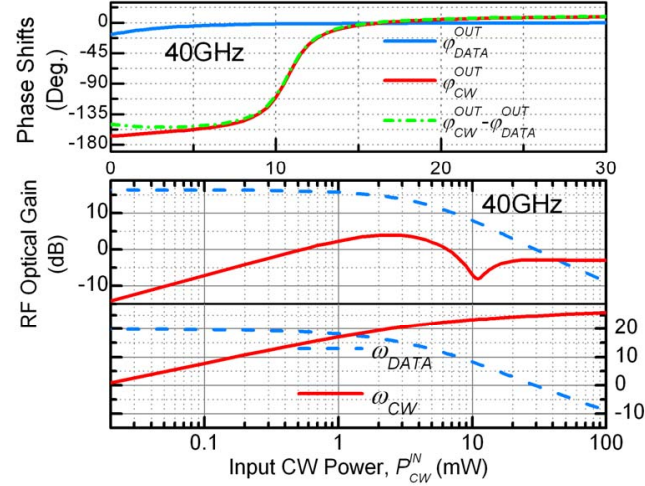

(a)

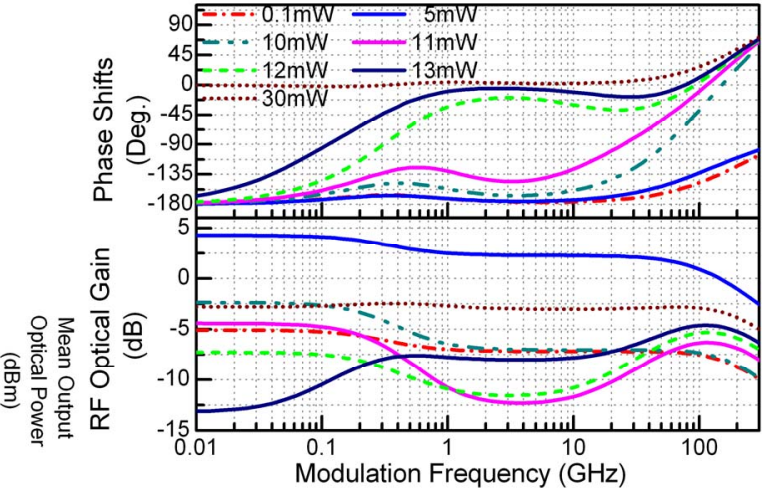

(b)

Fig. 4(a) Characteristics of the RF output signal at a modulation frequency of 40GHz based on XGM in QD SOAs. (Top) Phase shift. (Bottom) RF optical gain and mean output optical power as a function of input CW power. (b) Spectral characteristics of the XGM converted output signal of QD SOAs for different input CW power. (Top) Phase shift. (Bottom) RF optical gain. The input data signal is 1mW input with $20 \%$ modulation index. Injection current of $Q D S O A$ s is $10 \mathrm{kA} / \mathrm{cm}^{2}$.

\section{CONCLUSION}

We numerically demonstrate two types of microwave phase shifters based on CPO and XGM effects in QD SOAs at modulation frequencies much higher than the inverse of carrier lifetime. In both cases, ultrafast nonequilibrium inter-subband carrier dynamics in QDs plays an import role in extending the bandwidth to $100 \mathrm{GHz}$ or even higher.

\section{ACKNOWLEDGEMENTS}

The authors acknowledge support from the FP7 GOSPEL project financed by the European Commission, the QUEST project financed by the Danish Research Councils and the NATEC centre funded by VILLUM FONDEN.

\section{REFERENCES:}

[1] M.S. Bigelow, N.N. Lepeshkin, R.W. Boyd, Phys. Rev. Lett., 90, 113903, 2003.

[2] C. Chang-Hasnain and S.L. Chuang, J. Lightwave Technol., 24, 4642, 2006.

[3] J. Mørk, F. Öhman, M. van der Poel, Y. Chen, P. Lunnemann, K. Yvind, Laser Photonics Rev., 3, 30, 2009.

[4] A. Matsudaira et al., Opt. Lett., 32, 2894, 2007.

[5] A.P. Bogatov, P. G. Eliseev, P.G.Okhotnikov, M. P. Rakhval'skil, and K. A. Khairetdinov, Sov. J. Quantum Electron, 13, 1221 (1983).

[6] F. Öhman, K. Yvind, J. Mørk, IEEE Photon. Technol. Lett., 19, 1145, 2007.

[7] W. Xue, S. Sales, J. Capmany, and J. Mørk, Opt. Express, 18, 6156-6163, 2010.

[8] M.R. Fisher and S.L. Chuang, IEEE Photon. Technol. Lett., 18, 1714, 2006.

[9] M. Sugawara et al., J. Phys. D: Applied Phys., 38, 2126-2134, 2005.

[10] T. W. Berg, J. Mørk, IEEE J. Quantum Electron., 40, 1527, 2004.

[11] A.V. Uskov, E.P. O’Reilly, M. Laemmlin, N.N. Ledentsov, D. Bimberg, Opt. Commun., 248, 211, 2005.

[12] J. Kim, M. Laemmlin, C. Meuer, D. Bimberg, G. Eisenstein, IEEE J. Quantum Electron., 45, 240, 2009.

[13] D. Nielsen et al., Appl. Phys. Lett., 92, 211101, 2008.

[14] Y. Chen, J. Mørk, Opt. Lett., 35, 697, 2010

[15] Y. Chen, J. Mørk, Ultrahigh-frequency microwave phase shifts mediated by ultrafast dynamics in quantum dot semiconductor optical amplifiers, IEEE Photon. Technol. Lett., 2010 (In press).

[16] I. O’Driscoll, T. Piwonski, C.F. Schleussner, J. Houlihan, G. Huyet, R.J. Manning, Appl. Phys. Lett., 91, $071111,2007$.

[17] T.R. Nielsen, P. Gartner, F. Jahnke, A.A. Sawchuk, Phys. Rev. B, 69, 235314, 2004.

[18] T. Markussen, P. Kristensen, B. Tromborg, T. W. Berg, J. Mørk, Phys. Rev. B, 74, 195342, 2006.

[19] R. Heitz, H. Born, F. Guffarth, O. Stier, A. Schliwa, A. Hoffmann, D. Bimberg, Phys. Rev. B, 64, $241305(\mathrm{R}), 2001$.

[20] G. Bramann et al., IEEE. J. Quantum Electron., 41, 1260, 2005.

[21] M. Tessler, R. Nagar, G. Eisenstein, IEEE J. Quantum Electron., 28, 2242, 1992.

[22] N. Cheng, J. C. Cartledge, J. Lightwave Technol., 22, 1805, 2004. 